\title{
Theranostic Implications of Nanotechnology in Multiple Sclerosis: A Future Perspective
}

\author{
Ajay Vikram Singh, ${ }^{1,2}$ Manish Khare, ${ }^{3}$ W. N. Gade, ${ }^{1}$ and Paolo Zamboni ${ }^{4}$ \\ ${ }^{1}$ Department of Biotechnology, University of Pune, Ganeshkhind Road, Pune 411 007, India \\ ${ }^{2}$ Center for Biotechnology and Interdisciplinary Studies, Rensselaer Polytechnic Institute, Room 2145, 110 8th Street, \\ Troy, NY 12180, USA \\ ${ }^{3}$ Department of Applied Sciences, Maharashtra Academy of Engineering, Alandi (D), Pune 412 105, India \\ ${ }^{4}$ Centre for Vascular Disease, University of Ferrara, 41100 Ferrara, Italy \\ Correspondence should be addressed to Ajay Vikram Singh, ajaysingh@unipune.ac.in
}

Received 9 August 2012; Accepted 9 November 2012

Academic Editor: Pietro Invernizzi

Copyright (C) 2012 Ajay Vikram Singh et al. This is an open access article distributed under the Creative Commons Attribution License, which permits unrestricted use, distribution, and reproduction in any medium, provided the original work is properly cited.

\begin{abstract}
Multiple Sclerosis is a multifactorial disease with several pathogenic mechanisms and pathways. Successful MS management and medical care requires early accurate diagnosis along with specific treatment protocols based upon multifunctional nanotechnology approach. This paper highlights advances in nanotechnology that have enabled the clinician to target the brain and CNS in patient with multiple sclerosis with nanoparticles having therapeutic and imaging components. The multipartite theranostic (thera(py) + (diag)nostics) approach puts forth strong implications for medical care and cure in MS. The current nanotheranostics utilize tamed drug vehicles and contain cargo, targeting ligands, and imaging labels for delivery to specific tissues, cells, or subcellular components. A brief overview of nonsurgical nanorepair advances as future perspective is also described. Considering the potential inflammatory triggers in MS pathogenesis, a multifunctional nanotechnology approach will be needed for the prognosis.
\end{abstract}

\section{Introduction}

Multiple sclerosis (abbreviated MS), disseminated sclerosis, and encephalomyelitis disseminata are synonyms to an autoimmune condition rather than disease in which cells from immune lineage attack nervous system bringing demyelination $[1,2]$. It has passed more than a century since Charcot, Carswell, Cruveilhier, and others described the clinical and pathological characteristics of multiple sclerosis [3]. The onset of this enigmatic and progressive disorder of white matter of central nervous system (CNS) occurs in young age and is more common in females [4]. There are clinically defined MS patients with a prevalence that ranges between 2 and 150 per 100,000 [5]. High resolution magnetic resonance imaging (MRI) and spectroscopic analysis are fundamental tools for clinicians, assisting in prognosis of the disease [6]. This further helps in monitoring pathological progression and course of treatment of the disease. MS affects the ability of nerve cells in the brain and spinal cord to communicate with each other. Neurons communicate with each other by sending electrochemical signals called action potential along extending processes called axons, which are wrapped in an insulating lipoprotein, a dielectric substance called myelin $[7,8]$. In MS, body's own immune system attacks and damages the myelin. In abbreviated MS, the terms "scleroses" refers to scars, plaques, or lesions, those appear as "multiple" patches along white matter of cerebrospinal regions of the brain and spinal cord that can be seen in drawing from a Carswell book in 1838, the first clinical picture so far drawn $[7,8]$. Many studies have shown the mechanism involved in disease process, but causes are unknown though indicate multifactorial theories including genetics and immune infections as the central role [9]. Neurological symptoms with physical and cognitive disability; new symptoms occurring either in discrete attacks (relapsing forms) or slowly accumulating over time (progressive forms) are delineated factors to clinically assess the disease, but permanent neurological problems aggravate as the disease 


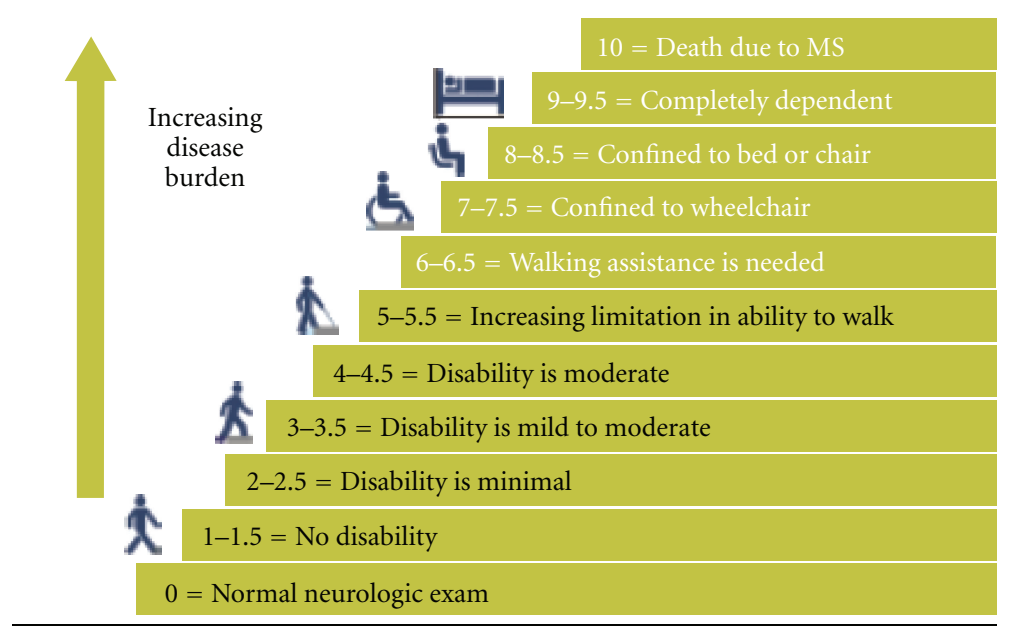

EDSS indicates expanded disability status scale; MS indicates multiple sclerosis.

FIGURE 1: Scaling the progression of disability. EDSS score in MS (published with permission of [11]).

advances [10]. The Kurtzke Expanded Disability Status Scale (EDSS) measures the progression of MS using a rating scale between 0 and 10 which defines disability status with progression of the diseases (Figure 1) [11]. The present articles give an overview of how nanotechnological implications can be utilized to improve quality of life (QoL) in MS patients which has been unrealized so far. We principally focus on theranostic approaches at nanoscale which has been successfully implemented in other CNS disorders (AD, PD, ALS, etc.) and open promising avenues in MS too $[12,13]$.

In this short perspective, we will first discuss nanotechnology-based therapeutic approaches utilizing nanoparticles as programmed drug delivery vehicle for neuroprotections and neuronal enhancement in diseased brain. Our discussion will be focused mainly on material design strategies circumventing to cross blood barrier and neurovasculature. Then, we represent an overview of advances made in nanodiagnostic assessment using NPs as contrast agent and/or multipartite system to deliver therapeutic + diagnostic (theranostic) together.

\section{Nanoscience and Technology in CNS Disorders}

In recent decades, nanotechnology has emerged as an impressive tool of treating neurological disease, with the radical changing the way we approach the CNS-targeted neurotherapeutics in the past. This lead to promising progress into treatments for diseases of the brain and CNS in spite of limited therapeutic options for many patients with neuropathology worldwide [14]. The major advantage of nanoscale technology supporting therapeutic application in neuropathology stems from nanoengineering and conjugation opportunities of therapeutic molecules with nanoparticles [15].

This in turn supports stability of drug molecule and helps to cross the blood-brain barrier for targeting specific cell signaling in brain. Particularly in nervous system where cells frequently loose the regenerative capacity following in vivo injury, nanomolecules are used as matrix to promote neural elongation and support cell survival in damaged cells or act as vehicles for gene delivery to tame molecular responses to endogenous pathological stimuli [16]. A wide variety of nanodevices and nanomaterials with capability to engineer the structure-function relationship matching with nanoscale molecular hierarchy in neuronal system make the nanotechnology powerful tool in treating the neurological disorders $[17,18]$.

\section{Blood-Brain Barrier (BBB) and Reticuloendothelial System (RES) in MS}

The brain barrier occurs along all capillaries and consists of tight junctions around the capillaries that do not exist in normal circulation at the base of the brain [16]. Endothelial cells restrict the diffusion of microscopic objects (e.g., bacteria) and large or hydrophilic molecules into the cerebrospinal fluid (CSF), while allowing the diffusion of small hydrophobic molecules $\left(\mathrm{O}_{2}, \mathrm{CO}_{2}\right.$, hormones) [19]. Cells of the barrier actively transport metabolic products such as glucose across the barrier with specific proteins (Figure 2). This barrier also includes a thick basement membrane and astrocytic end feet. The strong association of BBB in MS has been widely elucidated with histopathological and molecular changes $[20,21]$. The relapsing and progressive episodes in MS "attack" has shown the broken blood-brain barrier in a section of the brain or spinal cord, allowing $\mathrm{T}$ lymphocytes to cross over and attack the myelin which gradually leads to complete demyelination $[20,22]$. Our recent hypothesis also corroborates that a complex pattern of extracranial venous stenosis determines flow abnormalities such as reflux and blockages in the main extracranial outflow routes, namely, the internal jugulars and the azygos vein [23-25].

This creates a collateralization of the venous outflow with increased mean transit time and reduced perfusion of the brain parenchyma of MS patients [26]. Reduced perfusion 
(a) Neurovascular unit (blood-brain barrier)

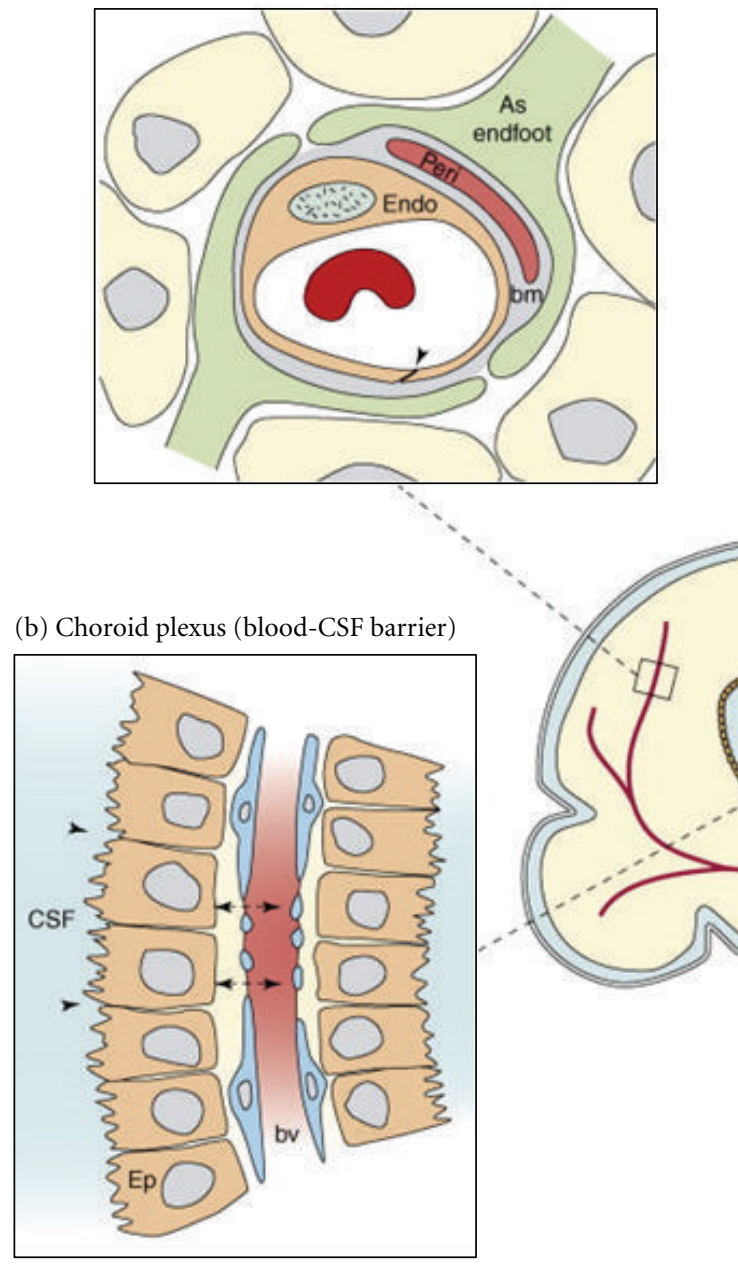

(c) Meninges (arachnoid barrier)

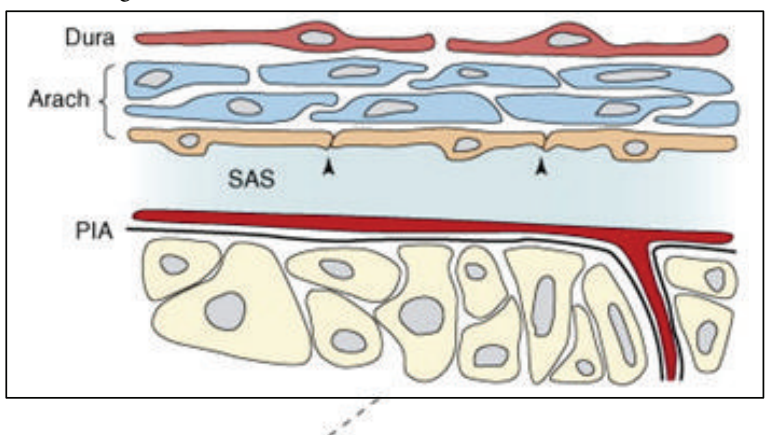

(d) Neuroependyma (fetal CSF-brain barrier)

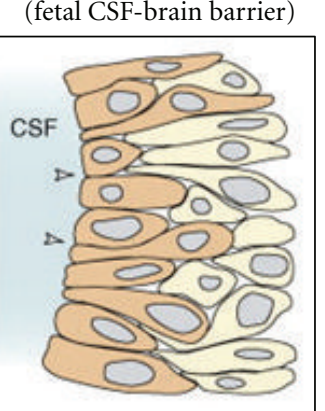

(e) Adult ependyma (free exchange)

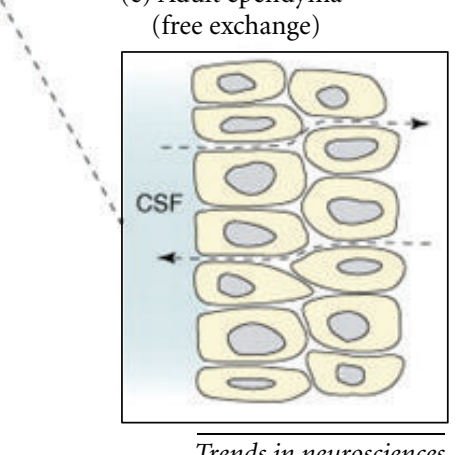

FIGURE 2: Barrier interfaces in brain. (a) endothelial cells (endo) in the neurovascular unit have luminal tight junctions (shown by the arrow) that form the physical barrier of the interendothelial cleft. Outside the endothelial cell is a basement membrane (bm) which also surrounds the pericytes (Peri). Around all of these structures are the astrocytic end-feet processes from nearby astrocytes. (b) The endothelial cells of choroid plexus blood vessels are fenestrated and form a nonrestrictive barrier (shown by dashed arrows) between the cerebrospinal fluid (CSF) and blood vessel (bv). The epithelial cells (ep) have apical tight junctions (shown by arrows) that restrict intercellular passage of molecules. (c) In the meninges, the blood vessels of the dura are fenestrated and provide little barrier function (not shown). However, the outer cells of the arachnoid membrane (Arach) have tight junctions (shown by arrows), and this cell layer forms the physical barrier between the CSF-filled subarachnoid space (SAS) and overlying structures. The blood vessels between the arachnoid membrane and the pial surface (PiA) have tight junctions (not shown). (d) In early development, the neuroependymal cells are connected to each other by strap junctions (shown by arrows) that are believed to form the physical barrier restricting the passage of larger molecules, such as proteins, but not smaller molecules, such as sucrose. (e) The mature adult ventricular ependyma does not restrict the exchange of molecules (shown by dotted arrows). The neurovascular unit (a), blood-CSF barrier (b), and arachnoid barrier (c) are common between developing and adult brain, whereas fetal neuroependyma (d) differs from adult ependyma (e) (cited from [19] with permission from cell press).

is a typical aspect of MS and cannot be explained of course by autoimmunity [27]. It might explain aspects of hypoxialike conditions in the MS plaques, early axon damage in absence of T-cells and oxidative stress with mitochondria impairment [28]. Another constant feature linked with hampered venous outflow is represented by blood-brain barrier breakdown. The latter may favor erythrocytes diapedesis with iron deposition into the brain parenchyma, which triggers a further local inflammatory response and amplifies the oxidative stress [29]. It will not be an exaggeration if, 
rather than being a disease of the immune system, MS is termed as a disease of the blood-brain barrier and RES [30]. This is followed by increased intravenous pressure, bloodbrain barrier breakdown, and iron deposition into the brain parenchyma, which triggers a local inflammatory response [30]. It will not be an exaggeration if, rather than being a disease of the immune system, MS is termed as a disease of the blood-brain barrier and RES [31].

\section{Drug Delivery System Crossing BBB}

Conjugation of therapeutic peptides or antibodies to the surface of magnetic nanomaterials helped in direct targeting and potential disruption of active signaling pathways of the tumor cell surface [32]. This field further opens potential avenues of the magnetic nanoparticles in translation studies in the brain pathology, such as imaging and targeting the sclerotic lesion with growth factor to treat the lesions in MS patients [33]. Delivery of conventional therapeutic to brain and CNS disorder represents a formidable challenge due to the presence of the blood-brain barrier and complex interplay of endothelial cells, astrocyte and pericytes (RES) at $\mathrm{BBB}$ in the normal brain [34]. The active targeting strategy with site-specific ligands binding increases penetration and surface nanoengineering of NPs, which provided new ways to control pharmacokinetics and bioavailability of CNSrelated drugs across BBB and RES [18, 35]. PEGylation of liposome and maintaining the particle diameter at $<100 \mathrm{~nm}$ help in combating problem associated with conventional liposomes (aggregation, short half-lives, modest transport capacity across the blood-brain barrier, and rapid RES clearance) by receptor or absorptive-mediated transcytosis [36]. Coating the liposome surface with monoclonal antibodies to glial fibrillary acidic proteins, transferrin receptors or human insulin receptors (nanoliposome) further help in escaping RES and $\mathrm{BBB}$ and delivering therapeutic genes $[37,38]$.

\section{Polymeric Artificial Cells}

Nanotechnology allows precise control over in vitro mimesis of molecular features at nanoscale for controlling materialcell interactions. This in turn induces specific developmental processes and cellular responses including differentiation, migration, and outgrowth in neuronal cells $[39,40]$. Inspired by polymeric artificial cells [41], hollow fibres or threedimensional polymeric structures as a capsule, protected from immune rejection by an artificial semipermeable membrane, have been made by macro- and microencapsulation $[13,42,43]$. The cell-loaded capsules can be implanted into the damaged brain area favoring the local, targeted, and long-term release of drugs or proteins [42, 44, 45]. The microcapsule loaded with ciliary neurotrophic factor(CNTF-) producing fibroblasts encapsulated into polymers with a vitrogen matrix and implanted intrathecally in clinical trial of amyotrophic lateral sclerosis in mouse (an animal model of MS) demonstrated in situ sustained delivery of CNTF without any immune- or cytotoxicity $[13,46]$.
However, results obtained in this trial are matter of further investigation to determine whether enhanced survival is secondary to the transplant environment and/or the epithelial cells fibroblasts [47].

\section{Carbon Base Nanomaterial}

Carbon nanotubes (CNTs) have electrical, mechanical, and chemical properties, and nanoscale features of CNTs make them better suited as an interface with neurons for stimulating and recording neural activity $[48,49]$. Notably, purified carbon nanotubes used as substrate/scaffolds reported to promote the formation of nanotube-neuron hybrid networks, able per se to trigger neuron integrative abilities, network connectivity, and synaptic plasticity [50, 51]. The stable interaction of carbon nanotube platforms with stem cell lineage sparked its versatile application in nerve tissue engineering to probe and augment cell behavior [50, 52]. It further opens new routes to treat CNS in MS pathology for nongenetic manipulations of neuronal performance and network signaling in vivo as demonstrated for contemporary disorders $[18,53]$.

\section{Polymeric Micelles and Nanoparticles}

Recent advances in nanoparticle design have demonstrated tremendous potential in engineering matrix chemistry of nanoparticles to design stimuli responsive polymeric nanocarriers [54].

Versatile strategies and protocols provide platform to tune intracellular stimulus (e.g., reducing nature of the cytosol compared with the extracellular space or the endosomal pH drop) [55] or to an external stimulus (e.g., applied magnetic field or exposure to a specific wavelength of light) $[56,57]$. The specific stimulus helps in triggering the drug release in situ via covalent bond cleavage between carrier (vehicle) and cargo (drug) at target (e.g., cell or tissue) $[58,59]$. The researches have designed "drug depots" with controlled release micelle-drug compositions. The core-shell architecture of amphiphilic block copolymers and micelle makes them particularly attractive for drug delivery vehicle [60]. The core can incorporate considerable amounts (up to $20 \%-30 \%$ weight) of water-insoluble drugs (Figure 3 ). Polymeric shell increases their pharmacokinetic release by preventing nonspecific interactions with enzymes, serum proteins, and nontarget cells. This further inhibits premature degradation and release of drug in dispersions, and the drug is released from the micelle via diffusion at specified target $[13,58]$. Inspired by fenestration in tumor, targeting brain tumor vasculature with circulating nanoparticles with inherent accessibility of vascular components during angiogenesis opens many perspectives in MS patients [61]. It involves complex interplay of upregulation and secretion of growth factors, which activates endothelial cells to secrete matrix metalloproteinase (MMPs) [62], which degrade the extracellular matrix (ECM) near the brain lesions [63]. This actively provides access to other cells to migrate at lesion site promoting ECM remodeling and cell proliferation $[64,65]$. Liposome containing therapeutic has been 


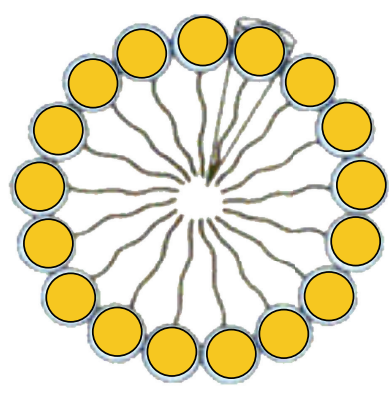

Micelles with hollow core
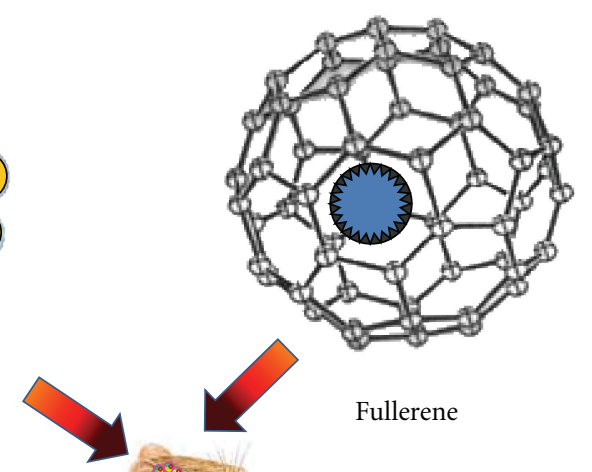

Fullerene

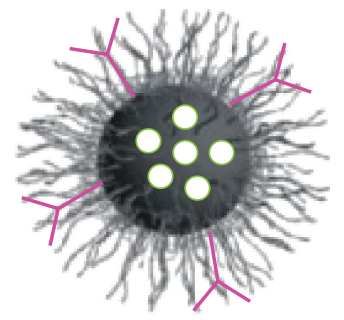

PEGylated liposome with the drug and $\mathrm{Ab}$ conjugates

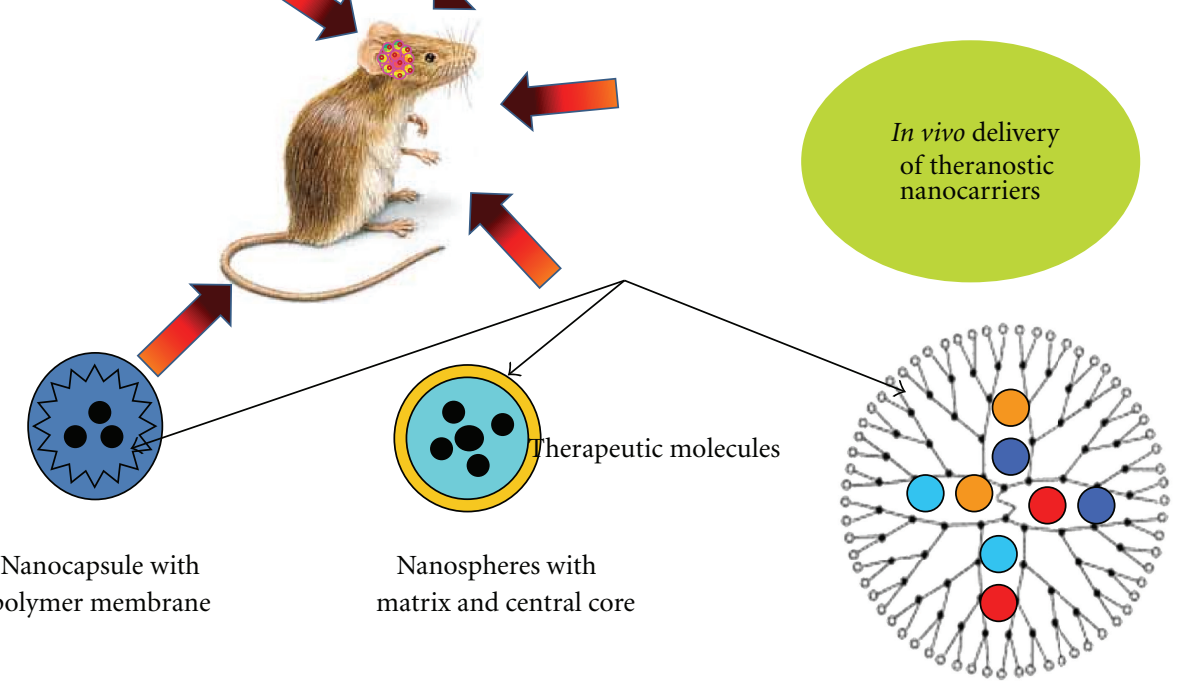

Figure 3: Polymeric nanocarriers in current CNS disorders. Micelle core is promising site for loading insoluble therapeutic agents, while liposome can be targeted by conjugating Ab linker. Fullerene cage surfaces can be functionalized for targeted delivery. Branched dendrimers and nanocapsules are potential nanotheranostic agents.

targeted to vasculature by the attachment of the arginineglycine-aspartic acid (RGD) peptide and demonstrated many marked fold improvement in drug efficacy compared with the free drug $[66,67]$.

\section{Emerging Concept of Nanoneuroprotection}

The aim of neuroprotection is to limit neuronal dysfunction/death after chronic CNS injury as happens in $\mathrm{AD}, \mathrm{PD}$, and MS, which results in salvage, recovery, or regeneration of the nervous system $[68,69]$. Many nanomaterials with antioxidant properties have shown the potential to eliminate reactive oxygen species (ROS) in the brain. Particularly, cerium and yttrium oxides $\left(\mathrm{CeO}_{2}\right.$ and $\left.\mathrm{Y}_{2} \mathrm{O}_{3}\right)$ NPs showed ROS mitigation in in vitro conditions using hippocampal neuronal cells $[13,70]$. Another class of novel nanomaterial receiving attention for neuroprotection is fullerene and its derivatives. The three-dimensional arrays of evenly spaced carbon atoms with high degree of unsaturated bonds display high reactivity with oxygen free radicals and possess antioxidant and free radical scavenger properties as water-soluble derivatives $[12,71]$. Glutamate receptors, which mitigate neuronal toxicity via intracellular calcium influx and limiting excitotoxicity, were shown by fullerenol [polyhydroxylated C60] and carboxyfullerene [malonic acid
C60 derivative], respectively, in an in vivo mouse model of familial amyotrophic lateral sclerosis, an animal model for MS [72, 73]. Here, fullerenols might partly inhibit glutamate receptors, as they had no effect on GABAA or taurine and/or lowered glutamate-induced elevations in intracellular calcium, which is an important mechanism of neuronal excitotoxicity involving receptors [74]. This opens a wide perspective and scope of fullerene derivatives in MS therapeutics and diagnosis (theranostics); nonetheless, lipid peroxidation, as well as decrease of glutathione in the gill cells, is a major concern considering it for neuroprotecting agent [68]. Compromising the toxicity of fullerenes with its potential theranostic applications in biomedicine, researchers found ways to modify CNTs and fullerene surfaces as demonstrated using single-walled CNTs (SWCNTs) and multiwalled (MWCNTs) via purification and chemical modification, aimed to increase solubility and decrease toxicity $[75,76]$.

Neuroinflammation is another hallmark of MS, and evidence from animal models suggests a mutual interplay of microglia, astrocyte, and $\mathrm{T}$ cells, other than demyelination [77]. In advance stages of MS, targeting neuroinflammation as potent therapeutic strategy has shown a promising strategy $[78,79]$. However, drug diffusion across BBB and neurovasculature of MS brain yet remain a major challenge. 
In a recent approach based on nanomaterials (polyamidoamine dendrimers), authors demonstrated targeted delivery of therapeutic localized in activated microglia and astrocytes of diseased brain which suppresses neuroinflammation and leads to a marked improvement in motor function $[15,80]$. The nanomaterial-based protocol in the current work provides an opportunity for clinical translation and opens window of opportunity for the treatment in advance MS pathology as successfully shown for animal models of MS $[78,81]$.

\section{Implications of Nanotechnology in Neurosurgery: Nanobodies Reaching MS Lesion Sites}

In the last decade, nanoscience and technology (NST) evolved as applied field beyond notion and speculations and spurred a strong impact not only in clinical sciences but also in almost all occupations of human reach [8284]. Particularly in neurobiology and clinical surgery, until recently, it proved to be sophisticated technique to enable micronanoscale cellular engineering and manipulations [85]. The nanosurgery will be the next medical frontier in neurobiology, which will eventually make substantial contributions to the advancement of neurosurgery in the near future $[86,87]$. Nanosurgery will involve nanoimaging and clearing of the defects/disorders at cellular and subcellular levels. Synthetic nanoscale magnetic materials (e.g., cytobots and karyobots) with impressive properties will promote regeneration in damaged axon and halt deleterious processes (e.g., hemorrhaging) via nanomanipulations [88, 89]. Nonsurgical nanorepairs and nanoneuromodulations will enable monitoring or stimulating diseased neurons. This will involve interaction of the nanobodies with lesions in nervous system in deep MS brain through electrical and/or electrochemical (e.g., neurotransmitter; $\mathrm{AcH}$ ) function by extending neuronal synaptic connections [90]. In the last decade, single-cell nanosurgery was thought to be neurophilosophy, but current tools such as QDs for nanoimaging, femtosecond pulses of near-infrared laser as surgical tweezers, multipartite NPs for neuromodulations, and AFM cantilever for nanomanipulations make it reality [91]. In one approach, researchers inserted AFM tip as sharp needle "nanoscissors" into the cell wall, which was indented by only one micrometer. This was much more delicate than routine clumsy method which is inevitably difficult to control in microcapillary procedures. AFM tip can be successfully inserted into the nucleus to and forth, and cell membrane quickly returns to its original shape [92]. This opens many perspectives for single-cell neurorepair such as coating tip with specific monoclonal antibodies (MAb) to interact with the intracellular protein traffic and enables monitoring the real-time intracellular chemistry. The ability to manipulate optically subcellular structures at submicrometer while minimizing photodamage has strong implications in MS brain lesions where neuronal cell contain a heterogeneous population of healthy and defective organelles. Improving the tools for single-cell and chromosomal nanosurgery and translating it into the cutting edge in vivo surgical device will revolutionize the field [93]. The concept of microrobotics and nanobodies with tiny magnetically driven spinning screws intended to swim along veins and carry drugs to infected tissues or even to burrow into diseased brain cells has risen from fiction to facts [93, 94]. In this series, the first Food and Drug Administration (FDA) approved, wirelessly controlled implantable microchip for osteoporosis drug delivery (e.g., human parathyroid hormone fragment (1-34) [hPTH(1-34)]) release device, is in market [95]. Also, the "camera in a pill" is one recent development which enabled surgeons to monitor real-time pathology and drug release in different accessible areas of gastrointestinal tracts (GITs) [96]. Research is underway to develop capsules with noninvasive propulsive and therapeutic capabilities in other realms of medicine to measure $\mathrm{pH}$, temperature, blood perfusion, and intestinal motility during its journey through the systemic circulation (Figure 4) [97].

\section{Diagnostic Implication of Nanomaterials for In Vitro and In Vivo Imaging (QDs, SPIONS, Gd)}

DNA-carrier gold nanoparticles- (AuNPs-) based biobarcode assay is competent in amplifying and detecting weak molecular signals up to attomolar concentration @sensitive protein biomarkers in CSF of diseased brain [98]. The bio-barcode assay is capable of amplifying and quantification of connecting molecular loop at ultralow concentration for signal detection, transduction (recording), and signal documentation at molecular scale [99]. These have strong implications in MS, since radio diagnoses (MRI, SWIP, Eco-doppler) are only available gold techniques for early detection of MS brain pathologies [100]. Another ultrasensitive and inexpensive optical method is localized surface plasmon resonance (LSPR) based upon anisotropic silver nanoparticles (AgNPs) nanosensor. The method relies on detecting perturbations in refractive index of the surrounding magnetic field which is an outcome of AgNPs-protein marker interactions at ultralow concentrations [101]. Atomic force microscopy has proven to be a useful technique in understanding molecular interactions at bionanointerfaces [102]. In a recent approach based on a scanning tunneling microscope (STM), authors demonstrated a sandwich-type immune binding assay with cantilever, which is sensitive to tip-to-biosurface interval. The signal transformation analyses give strong indication in the pulse-like peaks of tunneling current, and surrounding concentrations as low as $10 \mathrm{fg} / \mathrm{mL}$ can be detected [103]. A fast, ultra-sensitive, and specific nanosensor has been recently devised utilizing two-photon Rayleigh scattering signal emerging from bioconjugated tau protein AuNPs. The method claims to probe as low as $1 \mathrm{pg} / \mathrm{mL}$ within half an hour [104]. Furthermore, the implications multipartite NPs and QDs with multiple functionalities to treat ailed neurons have given hope for the future course of MS early diagnosis and cure (Figure 5). 


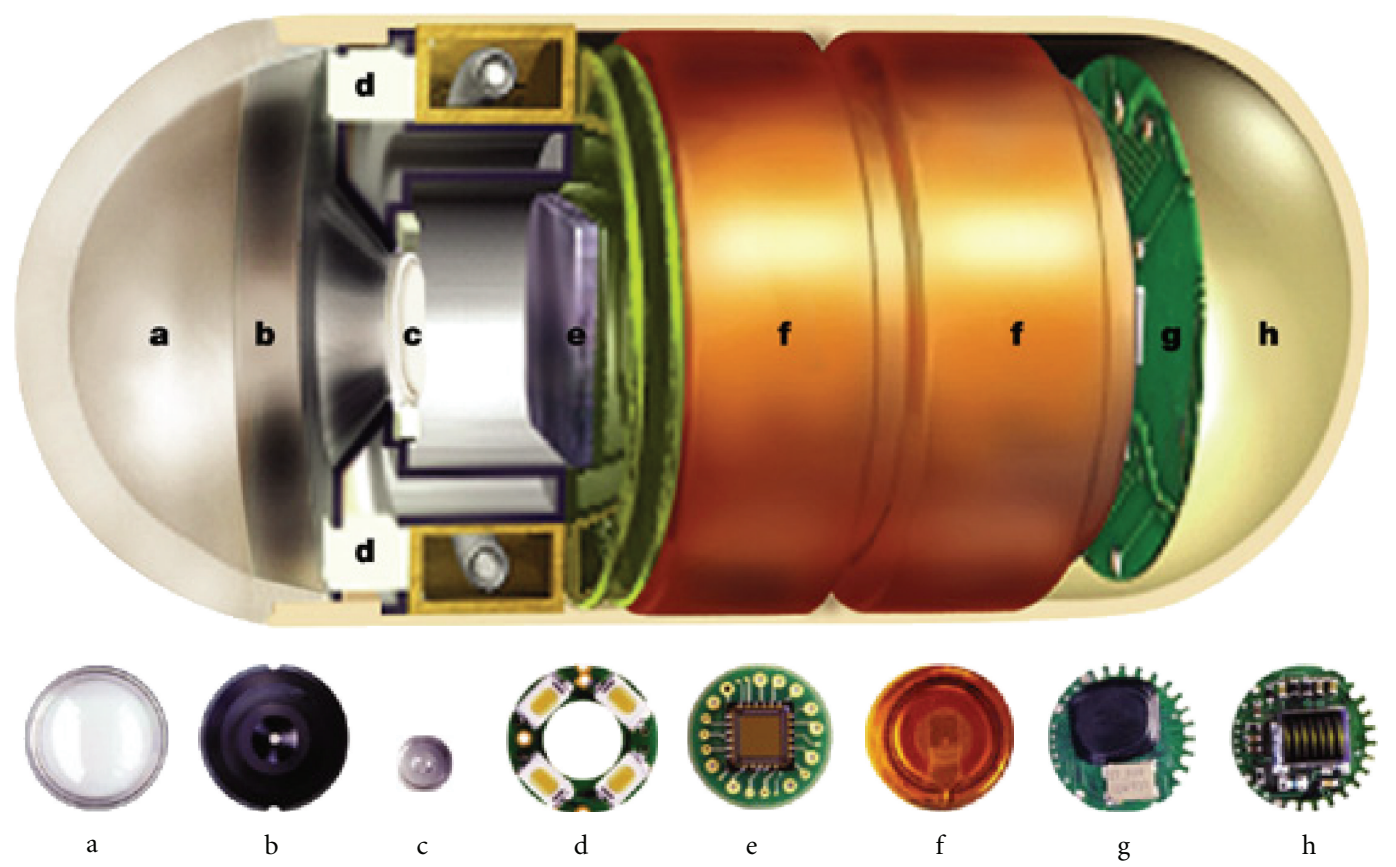

Figure 4: The M2A capsule camera. The device consists of a disposable plastic capsule that weighs 3.7 grams and measures $11 \mathrm{~mm}$ in diameter by $26 \mathrm{~mm}$ in length. The contents include an optical dome (a), a lens holder (b), a short focal-length lens (c), six white-light-emitting diode illumination sources $(\mathrm{d})$, complementary metal oxide silicon (CMOS) chip camera (e), two silver oxide batteries (f), a UHF band radio telemetry transmitter (g), and an antenna (h) (published with permission from [96]).

\section{New Contrast Agent for MRI to Detect Inflammatory Cellular Infiltration in MS}

Other than routinely used contrast imaging MRI, new and improved tools to image the cellular and metabolic features of MS are emerging rapidly. Gadolinium-DTPA (Gd-DTPA) is routinely used inflammatory marker in MS [105]. Recently, researchers demonstrated that ultrasmall superparamagnetic particles of iron oxide (USPIO) can visualize cellular infiltration and pluriformity of inflammation in MS more accurately compared to traditional techniques [106]. Interestingly, patterns of USPIO enhancement which have been observed contrary to routine Gd-DTPA exhibiting sensitivity and specificity of MRI in multiple sclerosis can be improved using USPIO (i) focal enhancement, (ii) ring-like enhancement, and (iii) return to isointensity of a previously hypointense lesion [107]. In rat model of ALS, lately, MRI has been utilized to follow labeled T cells with ultrasmall paramagnetic iron oxide (USPIO) NPs ex vivo and exhibited an infiltration of CD4+ lymphocyte in the midbrain/interbrain, while CD8+ cells were more confined to the brainstem region [108]. In another work, Machtoub et al. had successfully shown molecular imaging of brain lipid environment of lymphocytes in ALS mouse model using MRI and SECARS microscopy. They were able to detect the pathological regions in ALS rat brain via intravenously injected USPIO NP conjugated with anti-CD4 antibodies [109].

Micro- and nanoparticle technology in particular boosted MRI imaging as contrast agent tremendously [110].
It provide a novel opportunity of incorporating multiple functionalities into a single delivery vehicle, and reports show that, when combined with photoacoustic tomography (PAT), MRI sensitivity increases to picomolar concentration [111].

\section{Concluding Remark}

Albeit applications of nanotechnologies in neurological disorder treatment are in infancy; the potential of using these nanomaterials for treatment and diagnosis of multiple sclerosis opens many promises. Early MS therapy and diagnosis with traditionally means met with limited success outside of a few oral pharmacological agents capable of modifying anti-immune symptoms (e.g., Natalizumab) or physical exercise ameliorating motor dysfunctions. However, early diagnosis of MS would be the best approach in order to prevent irreversible and uncontrollable disability consequence. Many molecular markers and radiodiagnosis approaches successfully demonstrate the disease progress, but sensitivity to recognize onset of the stages achieved recently bases upon nanomaterials contrasts agents base. The nanomaterials nanodiagnostic tools utilize different nanoparticles/nanostructures and are based on different physicochemical interactions that may be utilized either in vitro or in vivo. Nevertheless, there are still many challenges regarding the immuno/geno/cytotoxicity of nanoparticles and micronanodevices especially in a complex biological milieu like brain with complex network of neuronal cells. 


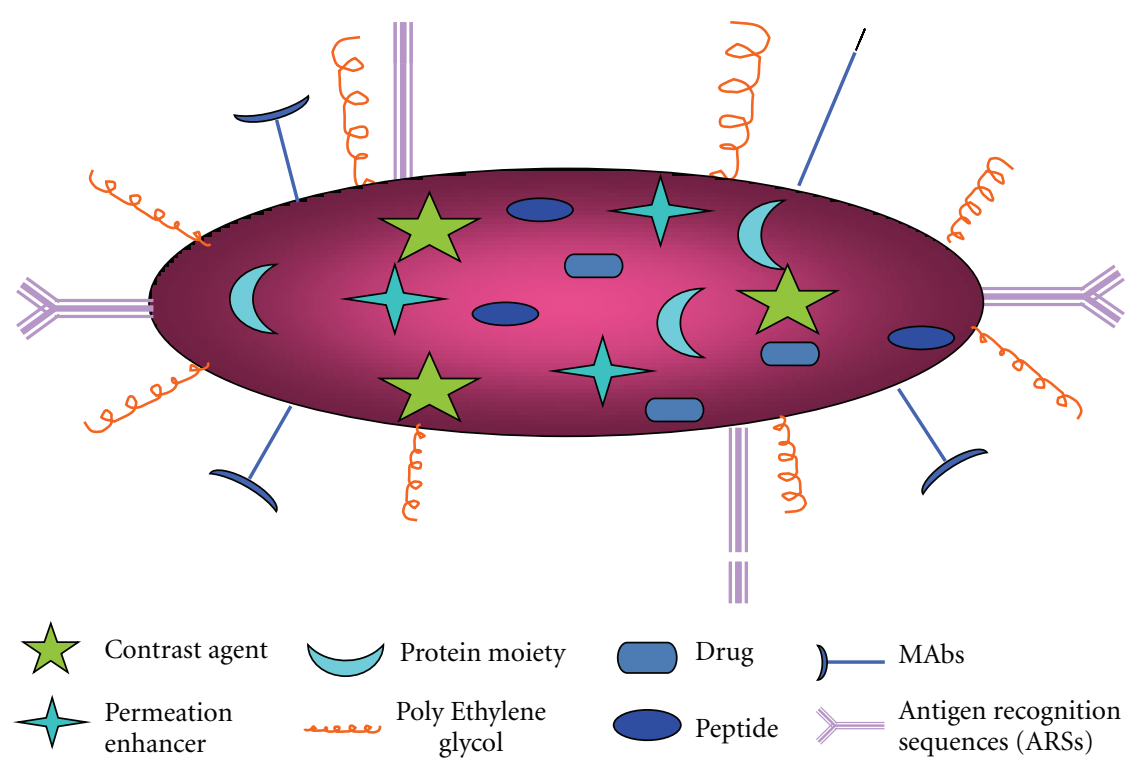

(a)

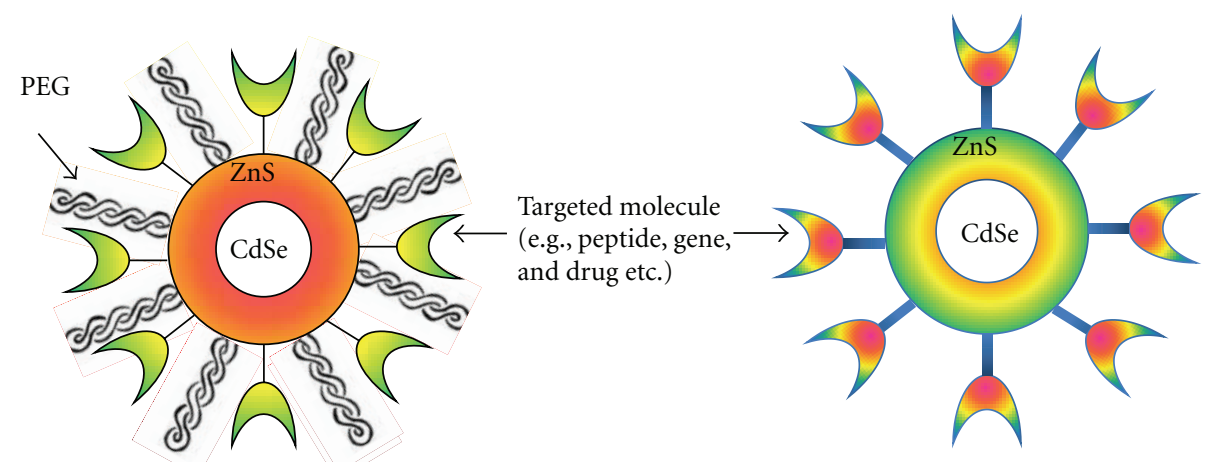

(b)
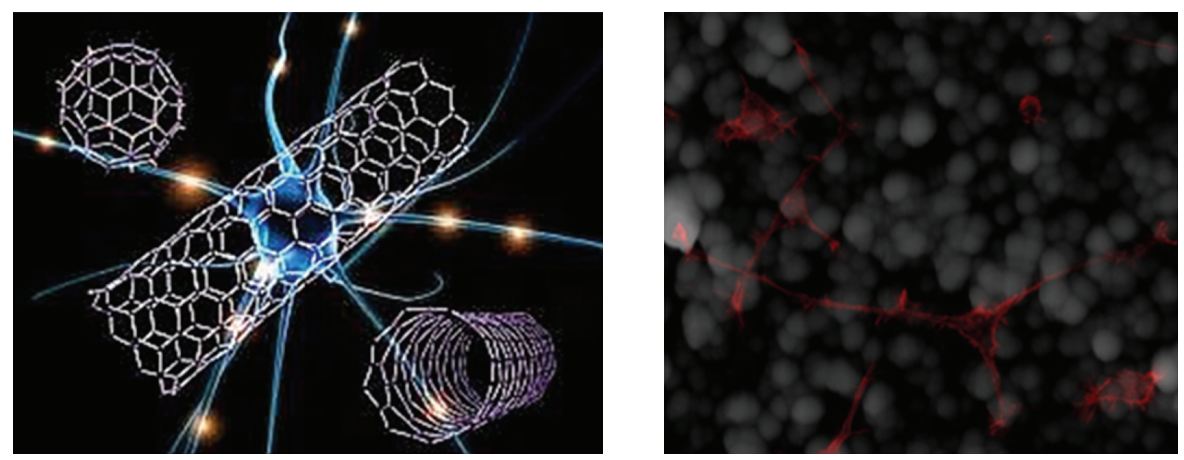

(c)

FIGURE 5: Multivariate nanocarriers. (a) multipartite nanostructure with targeting agent, permeation enhancer for BBB and RES, and multicomponent theranostic agent. PEGylation strategy for QDs for increasing uptake and combating BBB (b). (c) demonstrates interaction of neurons with the CNTs and fluorescently labelled neurons interaction with nanostructured surfaces.

Yet, a long and puzzling path is ahead to make the envisioned nanoneurosurgical approaches of curing MS diseases as a practical technology and, eventually, a routine clinical practice. The advances in biological microelectromechanical system (Bio-MEMS) and nanoelectromechanical system
(NEMS) need to be explored as surgical tool to target and cross the $\mathrm{BBB} / \mathrm{RES}$ to reach MS brain and perform requisite surgery. Moreover, with inherent complexity of the brain itself and the myriad cellular biochemical responses associated with injury and repair, we need to develop a highly 
interdisciplinary approach at the biotic/abiotic interface for recognition of disorder, neuroprotection, and neurorepair at the onset of MS. With the significant increases in the prevalence and incidence of MS and related disorders worldwide, the new approaches and interdisciplinary advances are very much needed to fight against the debilitating disorder.

\section{Acknowledgments}

The authors would like to dedicate this paper to late Rajiv Char who left them on 28-02-2010 after a long battle with MS. Department of Science and Technology (DST) is greatly acknowledged for the financial support under DST-PURSE Project.

\section{References}

[1] A. Comston and A. Coles, "Multiple sclerosis," The Lancet, vol. 359, no. 9313, pp. 1221-1231, 2002.

[2] J. A. Bobholz and S. Gremley, "Multiple sclerosis and other demyelinating disorders," The Little Black Book of Neuropsychology, pp. 647-661, 2011.

[3] K. J. Smith and W. I. McDonald, "The pathophysiology of multiple sclerosis: the mechanisms underlying the production of symptoms and the natural history of the disease," Philosophical Transactions of the Royal Society B, vol. 354, no. 1390, pp. 1649-1673, 1999.

[4] M. Debouverie, S. Pittion-Vouyovitch, S. Louis, and F. Guillemin, "Natural history of multiple sclerosis in a population-based cohort," European Journal of Neurology, vol. 15, no. 9, pp. 916-921, 2008.

[5] C. A. Jones, S. L. Pohar, S. Warren, K. V. Turpin, and K. G. Warren, "The burden of multiple sclerosis: a community health survey," Health and Quality of Life Outcomes, vol. 6, article 1, 2008.

[6] F. Manfredini, A. M. Malagoni, S. Mandini et al., "Nearinfrared spectroscopy assessment following exercise training in patients with intermittent claudication and in untrained healthy participants," Vascular and Endovascular Surgery, vol. 46, no. 4, pp. 315-324, 2012.

[7] B. Hemmer, J. J. Archelos, and H. P. Hartung, "New concepts in the immunopathogenesis of multiple sclerosis," Nature Reviews Neuroscience, vol. 3, no. 4, pp. 291-301, 2002.

[8] P. B. Carrieri, M. Petracca, S. Montella, M. Delfino, C. Sepe, and A. Gattoni, "Multiple sclerosis and systemic sclerosis: efficacy of interferon beta on skin lesions," Annals of the Rheumatic Diseases, vol. 67, no. 8, pp. 1192-1193, 2008.

[9] A. Ascherio and K. L. Munger, "Environmental risk factors for multiple sclerosis. Part I: the role of infection," Annals of Neurology, vol. 61, no. 4, pp. 288-299, 2007.

[10] F. D. Lublin and S. C. Reingold, "Defining the clinical course of multiple sclerosis: results of an international survey," Neurology, vol. 46, no. 4, pp. 907-911, 1996.

[11] J. F. Kurtzke, "Rating neurologic impairment in multiple sclerosis: an expanded disability status scale (EDSS)," Neurology, vol. 33, no. 11, pp. 1444-1452, 1983.

[12] G. A. Silva, "Neuroscience nanotechnology: progress, opportunities and challenges," Nature Reviews Neuroscience, vol. 7, no. 1, pp. 65-74, 2006.
[13] G. Orive, E. Anitua, J. L. Pedraz, and D. F. Emerich, "Biomaterials for promoting brain protection, repair and regeneration," Nature Reviews Neuroscience, vol. 10, no. 9, pp. 682692, 2009.

[14] L. N. Lin, Q. Liu, L. Song, F. F. Liu, and J. X. Sha, "Recent advances in nanotechnology based drug delivery to the brain," Cytotechnology, vol. 62, no. 5, pp. 377-380, 2010.

[15] J. R. Kanwar, X. Sun, V. Punj et al., "Nanoparticles in the treatment and diagnosis of neurological disorders: untamed dragon with fire power to heal," Nanomedicine, vol. 8, no. 4, pp. 399-414, 2012.

[16] E. A. Neuwelt, B. Bauer, C. Fahlke et al., "Engaging neuroscience to advance translational research in brain barrier biology," Nature Reviews Neuroscience, vol. 12, no. 3, pp. 169182, 2011.

[17] M. Srikanth and J. A. Kessler, "Nanotechnology-novel therapeutics for CNS disorders," Nature Reviews Neurology, vol. 8, no. 6, pp. 307-318, 2012.

[18] M. Fazil, B. S. Shadab, J. K. Sahni, and J. Ali, "Nanotherapeutics for Alzheimer's disease (AD): past, present and future," Journal of Drug Targeting, vol. 20, no. 2, pp. 97-113, 2012.

[19] N. R. Saunders, C. J. Ek, M. D. Habgood, and K. M. Dziegielewska, "Barriers in the brain: a renaissance?" Trends in Neurosciences, vol. 31, no. 6, pp. 279-286, 2008.

[20] W. M. Pardridge, "Molecular biology of the blood-brain barrier," Molecular Biotechnology, vol. 30, no. 1, pp. 57-69, 2005.

[21] T. Zeis, A. Probst, A. J. Steck et al., "Molecular changes in white matter adjacent to an active demyelinating lesion in early multiple sclerosis," Brain Pathology, vol. 19, no. 3, pp. 459-466, 2009.

[22] P. Zamboni, "The Big Idea: iron-dependent inflammation in venous disease and proposed parallels in multiple sclerosis," Journal of the Royal Society of Medicine, vol. 99, no. 11, pp. 589-593, 2006.

[23] P. Zamboni, R. Galeotti, E. Menegatti et al., "Chronic cerebrospinal venous insufficiency in patients with multiple sclerosis," Journal of Neurology, Neurosurgery and Psychiatry, vol. 80, no. 4, pp. 392-399, 2009.

[24] A. Laupacis, E. Lillie, A. Dueck et al., "Association between chronic cerebrospinal venous insufficiency and multiple sclerosis: a meta-analysis," CMAJ, vol. 183, no. 16, pp. E1203E1212, 2011.

[25] R. Zivadinov, M. Ramanathan, K. Dolic et al., "Chronic cerebrospinal venous insufficiency in multiple sclerosis: diagnostic, pathogenetic, clinical and treatment perspectives," Expert Review of Neurotherapeutics, vol. 11, no. 9, pp. 12771294, 2011.

[26] P. Zamboni, E. Menegatti, B. Weinstock-Guttman et al., "Hypoperfusion of brain parenchyma is associated with the severity of chronic cerebrospinal venous insufficiency in patients with multiple sclerosis: a cross-sectional preliminary report," BMC Medicine, vol. 9, article 22, 2011.

[27] M. D’haeseleer, M. Cambron, L. Vanopdenbosch, and J. De Keyser, "Vascular aspects of multiple sclerosis," The Lancet Neurology, vol. 10, no. 7, pp. 657-666, 2011.

[28] A. P. D. Henderson, M. H. Barnett, J. D. E. Parratt, and J. W. Prineas, "Multiple sclerosis: distribution of inflammatory cells in newly forming lesions," Annals of Neurology, vol. 66, no. 6, pp. 739-753, 2009.

[29] S. M. LeVine and A. Chakrabarty, "The role of iron in the pathogenesis of experimental allergic encephalomyelitis 
and multiple sclerosis," Annals of the New York Academy of Sciences, vol. 1012, pp. 252-266, 2004.

[30] A. V. Singh and P. Zamboni, "Anomalous venous blood flow and iron deposition in multiple sclerosis," Journal of Cerebral Blood Flow and Metabolism, vol. 29, no. 12, pp. 1867-1878, 2009.

[31] T. G. D’Aversa et al., "Mylin basic protein induces inflamattory mediatirs from primary human endothelial cells and blood brain barrier disruption: implications of the multiple sclerosis," Neuropathology and Applied Neurobiology. In press.

[32] M. Wankhede, A. Bouras, M. Kaluzova, and C. G. Hadjipanayis, "Magnetic nanoparticles: an emerging technology for malignant brain tumor imaging and therapy," Expert Review of Clinical Pharmacology, vol. 5, no. 2, pp. 173-186, 2012.

[33] G. De Rosa, G. Salzano, M. Caraglia, and A. Abbruzzese, "Nanotechnologies: a strategy to overcome blood-brain barrier," Current Drug Metabolism, vol. 13, no. 1, pp. 61-69, 2012.

[34] G. A. Silva, "Nanotechnology approaches to crossing the blood-brain barrier and drug delivery to the CNS," BMC Neuroscience, vol. 9, supplement 3, article S4, 2008.

[35] D. R. Siwak, A. M. Tari, and G. Lopez-Berestein, "The potential of drug-carrying immunoliposomes as anticancer agents," Clinical Cancer Research, vol. 8, no. 4, pp. 955-956, 2002.

[36] T. Patel, J. Zhou, J. M. Piepmeier, and W. M. Saltzman, "Polymeric nanoparticles for drug delivery to the central nervous system," Advanced Drug Delivery Reviews, vol. 64, no. 7, pp. 701-705, 2012.

[37] V. Rivest, A. Phivilay, C. Julien et al., "Novel liposomal formulation for targeted gene delivery," Pharmaceutical Research, vol. 24, no. 5, pp. 981-990, 2007.

[38] N.-F. Sun, Q.-Y. Meng, A.-L. Tian et al., "Nanoliposomemediated FL/TRAIL double-gene therapy for colon cancer: in vitro and in vivo evaluation," Cancer Letters, vol. 315, no. 1, pp. 69-77, 2012.

[39] A. V. Singh, C. Lenardi, L. Gailite, A. Gianfelice, and P. Milani, "A simple lift-off-based patterning method for microand nanostructuring of functional substrates for cell culture," Journal of Micromechanics and Microengineering, vol. 19, no. 11, Article ID 115028, 2009.

[40] A. V. Singh, L. Gailite, V. Vyas et al., "Rapid prototyping of nano- and micro-patterned substrates for the control of cell neuritogenesis by topographic and chemical cues," Materials Science and Engineering C, vol. 31, no. 5, pp. 892-899, 2011.

[41] T. M. Swi Chang, "Therapeutic applications of polymeric artificial cells," Nature Reviews Drug Discovery, vol. 4, no. 3, pp. 221-235, 2005.

[42] G. Orive, R. M. Hernández, A. R. Gascón et al., "Cell encapsulation: promise and progress," Nature Medicine, vol. 9, no. 1, pp. 104-107, 2003.

[43] S. Knippenberg, N. Thau, R. Dengler, T. Brinker, and S. Petri, "Intracerebroventricular injection of encapsulated human mesenchymal cells producing glucagon-like peptide 1 prolongs survival in a mouse model of als," PLoS ONE, vol. 7, no. 6, Article ID e36857, 2012.

[44] R. Fernandes and D. H. Gracias, "Self-folding polymeric containers for encapsulation and delivery of drugs," Advanced Drug Delivery Reviews, vol. 64, no. 14, pp. 1579-1589, 2012.

[45] S. Ausländer, M. Wieland, and M. Fussenegger, "Smart medication through combination of synthetic biology and cell microencapsulation," Metabolic Engineering, vol. 14, no. 3, pp. 252-260, 2012.

[46] P. Aebischer, M. Schluep, N. Déglon et al., "Intrathecal delivery of CNTF using encapsulated genetically modified xenogeneic cells in amyotrophic lateral sclerosis patients," Nature Medicine, vol. 2, no. 6, pp. 696-699, 1996.

[47] D. F. Emerich, G. Orive, and C. Borlongan, "Tales of biomaterials, molecules, and cells for repairing and treating brain dysfunction," Current Stem Cell Research and Therapy, vol. 6, no. 3, pp. 171-189, 2011.

[48] E. B. Malarkey and V. Parpura, "Carbon nanotubes in neuroscience," in Brain Edema XIV, Z. Czernicki et al., Ed., pp. 337-341, Springer, Vienna, Austria, 2010.

[49] G. Modi, V. Pillay, and Y. E. Choonara, "Advances in the treatment of neurodegenerative disorders employing nanotechnology," Annals of the New York Academy of Sciences, vol. 1184, pp. 154-172, 2010.

[50] W. Lee and V. Parpura, "Chapter 6-carbon nanotubes as substrates/scaffolds for neural cell growth," Progress in Brain Research, vol. 180, pp. 110-125, 2009.

[51] G. Cellot, E. Cilia, S. Cipollone et al., "Carbon nanotubes might improve neuronal performance by favouring electrical shortcuts," Nature Nanotechnology, vol. 4, no. 2, pp. 126-133, 2009.

[52] Y.-J. Huang, H.-C. Wu, N.-H. Tai, and T.-W. Wang, "Carbon nanotube rope with electrical stimulation promotes the differentiation and maturity of neural stem cells," Small, vol. 8, no. 18, pp. 2869-2877, 2012.

[53] A. Nunes, K. T. Al-Jamal, and K. Kostarelos, “Therapeutics, imaging and toxicity of nanomaterials in the central nervous system," Journal of Controlled Release, vol. 161, no. 2, pp. 290 306, 2012.

[54] L. Qiu, C. Zheng, Y. Jin, and K. Zhu, "Polymeric micelles as nanocarriers for drug delivery," Expert Opinion on Therapeutic Patents, vol. 17, no. 7, pp. 819-830, 2007.

[55] R. Tang, W. Ji, and C. Wang, "PH-responsive micelles based on amphiphilic block copolymers bearing ortho ester pendants as potential drug carriers," Macromolecular Chemistry and Physics, vol. 212, no. 11, pp. 1185-1192, 2011.

[56] Z. Zhu, E. Senses, P. Akcora, and S. A. Sukhishvili, "Programmable light-controlled shape changes in layered polymer nanocomposites," ACS Nano, vol. 6, no. 4, pp. 3152-3162, 2012.

[57] J. Ouyang, C. W. Chu, C. R. Szmanda, L. Ma, and Y. Yang, "Programmable polymer thin film and non-volatile memory device," Nature Materials, vol. 3, no. 12, pp. 918-922, 2004.

[58] R. A. Petros and J. M. Desimone, "Strategies in the design of nanoparticles for therapeutic applications," Nature Reviews Drug Discovery, vol. 9, no. 8, pp. 615-627, 2010.

[59] H. Rosen and T. Abribat, "The rise and rise of drug delivery," Nature Reviews Drug Discovery, vol. 4, no. 5, pp. 381-385, 2005.

[60] X.-B. Xiong, A. Falamarzian, S. M. Garg, and A. Lavasanifar, "Engineering of amphiphilic block copolymers for polymeric micellar drug and gene delivery," Journal of Controlled Release, vol. 155, no. 2, pp. 248-261, 2011.

[61] E. Ruoslahti, S. N. Bhatia, and M. J. Sailor, "Targeting of drugs and nanoparticles to tumors," Journal of Cell Biology, vol. 188, no. 6, pp. 759-768, 2010.

[62] A. V. Singh, L. Subhashree, P. Milani, D. Gemmati, and P. Zamboni, "Interplay of iron metallobiology, metalloproteinases, and FXIII, and role of their gene variants in venous leg ulcer," International Journal of Lower Extremity Wounds, vol. 9, no. 4, pp. 166-179, 2010. 
[63] T. J. Seabrook, A. Littlewood-Evans, V. Brinkmann, B. Pöllinger, C. Schnell, and P. C. Hiestand, "Angiogenesis is present in experimental autoimmune encephalomyelitis and pro-angiogenic factors are increased in multiple sclerosis lesions," Journal of Neuroinflammation, vol. 7, article 95, 2010.

[64] J. E. Holley, J. Newcombe, J. L. Whatmore, and N. J. Gutowski, "Increased blood vessel density and endothelial cell proliferation in multiple sclerosis cerebral white matter," Neuroscience Letters, vol. 470, no. 1, pp. 65-70, 2010.

[65] J. Van Horssen, C. D. Dijkstra, and H. E. De Vries, "The extracellular matrix in multiple sclerosis pathology," Journal of Neurochemistry, vol. 103, no. 4, pp. 1293-1301, 2007.

[66] D. Neri and R. Bicknell, "Tumour vascular targeting," Nature Reviews Cancer, vol. 5, no. 6, pp. 436-446, 2005.

[67] G. Von Maltzahn, J.-H. Park, K. Y. Lin et al., "Nanoparticles that communicate in vivo to amplify tumour targeting," Nature Materials, vol. 10, no. 7, pp. 545-552, 2011.

[68] B. Uttara, A. V. Singh, P. Zamboni, and R. T. Mahajan, "Oxidative stress and neurodegenerative diseases: a review of upstream and downstream antioxidant therapeutic options," Current Neuropharmacology, vol. 7, no. 1, pp. 65-74, 2009.

[69] R. J. M. Franklin and C. Ffrench-Constant, "Remyelination in the CNS: from biology to therapy," Nature Reviews Neuroscience, vol. 9, no. 11, pp. 839-855, 2008.

[70] D. Schubert, R. Dargusch, J. Raitano, and S. W. Chan, "Cerium and yttrium oxide nanoparticles are neuroprotective," Biochemical and Biophysical Research Communications, vol. 342, no. 1, pp. 86-91, 2006.

[71] L. Zhang, D. Alizadeh, and B. Badie, "Carbon nanotube uptake and toxicity in the brain," Methods in Molecular Biology, vol. 625, pp. 55-65, 2010.

[72] L. L. Dugan, D. M. Turetsky, C. Du et al., "Carboxyfullerenes as neuroprotective agents," Proceedings of the National Academy of Sciences of the United States of America, vol. 94, no. 17, pp. 9434-9439, 1997.

[73] S. S. Ali, J. I. Hardt, and L. L. Dugan, "SOD Activity of carboxyfullerenes predicts their neuroprotective efficacy: a structure-activity study," Nanomedicine, vol. 4, no. 4, pp. 283-294, 2008.

[74] L. L. Dugan, E. G. Lovett, K. L. Quick, J. Lotharius, T. T. Lin, and K. L. O'Malley, "Fullerene-based antioxidants and neurodegenerative disorders," Parkinsonism and Related Disorders, vol. 7, no. 3, pp. 243-246, 2001.

[75] A. Tan, L. Yildirimer, J. Rajadas, H. De La Peña, G. Pastorin, and A. Seifalian, "Quantum dots and carbon nanotubes in oncology: a review on emerging theranostic applications in nanomedicine," Nanomedicine, vol. 6, no. 6, pp. 1101-1114, 2011.

[76] S. R. Shin, H. Bae, J. M. Cha et al., "Carbon nanotube reinforced hybrid microgels as scaffold materials for cell encapsulation," ACS Nano, vol. 6, no. 1, pp. 362-372, 2012.

[77] R. W. Motl and L. A. Pilutti, "The benefits of exercise training in multiple sclerosis," Nature Reviews Neurology, vol. 8, no. 9, pp. 487-497, 2012.

[78] S. Kannan, H. Dai, R. S. Navath et al., "Dendrimer-based postnatal therapy for neuroinflammation and cerebral palsy in a rabbit model," Science Translational Medicine, vol. 4, no. 130, Article ID 130ra46, 2012.

[79] P. J. Gaillard, C. C. M. Appeldoorn, J. Rip et al., "Enhanced brain delivery of liposomal methylprednisolone improved therapeutic efficacy in a model of neuroinflammation," Journal of Controlled Release, vol. 164, no. 3, pp. 364-369, 2012.
[80] H. Dai, R. S. Navath, B. Balakrishnan et al., "Intrinsic targeting of inflammatory cells in the brain by polyamidoamine dendrimers upon subarachnoid administration," Nanomedicine, vol. 5, no. 9, pp. 1317-1329, 2010.

[81] A. R. Menjoge, R. M. Kannan, and D. A. Tomalia, "Dendrimer-based drug and imaging conjugates: design considerations for nanomedical applications," Drug Discovery Today, vol. 15, no. 5-6, pp. 171-185, 2010.

[82] A. V. Singh, A. Rahman, N. V. G. Sudhir Kumar et al., "Bioinspired approaches to design smart fabrics," Materials and Design, vol. 36, pp. 829-839, 2012.

[83] A. V. Singh, A.S. Ajay, N. Aditi et al., "Nanomaterials: new generation therapeutics in wound healing and tissue repair," Current Nanoscience, vol. 6, pp. 577-586, 2010.

[84] A. V. Singh, S. Maheshwari, D. Giovanni et al., "Nanoengineering approaches to design advanced dental materials for clinical applications," Journal of Bionanoscience, vol. 4, no. 12, pp. 53-65, 2010.

[85] S. Sant, S. L. Tao, O. Z. Fisher, Q. Xu, N. A. Peppas, and A. Khademhosseini, "Microfabrication technologies for oral drug delivery," Advanced Drug Delivery Reviews, vol. 64, no. 6, pp. 496-507, 2012.

[86] V. Kohli and A. Y. Elezzabi, "Prospects and developments in cell and embryo laser nanosurgery," Wiley Interdisciplinary Reviews, vol. 1, no. 1, pp. 11-25, 2009.

[87] M. Ebbesen and T. G. Jensen, "Nanomedicine: techniques, potentials, and ethical implications," Journal of Biomedicine and Biotechnology, vol. 2006, Article ID 51516, 11 pages, 2006.

[88] A. M. Khawaja, "The legacy of nanotechnology: revolution and prospects in neurosurgery," International Journal of Surgery, vol. 9, no. 8, pp. 608-614, 2011.

[89] R. A. Freitas, "Nanotechnology, nanomedicine and nanosurgery," International Journal of Surgery, vol. 3, no. 4, pp. 243-246, 2005.

[90] G. J. Tserevelakis, S. Psycharakis, B. Resan et al., "Femtosecond laser nanosurgery of sub-cellular structures in HeLa cells by employing Third Harmonic Generation imaging modality as diagnostic tool," Journal of Biophotonics, vol. 5, no. 2, pp. 200-207, 2012.

[91] G. D. M. Jeffries, J. S. Edgar, Z. Yiqiong, J. P. Shelby, F. Christine, and D. T. Chiu, "Using polarization-shaped optical vortex traps for single-cell nanosurgery," Nano Letters, vol. 7, no. 2, pp. 415-420, 2007.

[92] I. Obataya, C. Nakamura, S. Han, N. Nakamura, and J. Miyake, "Nanoscale operation of a living cell using an atomic force microscope with a nanoneedle," Nano Letters, vol. 5, no. 1, pp. 27-30, 2005.

[93] J. Wang and W. Gao, "Nano/microscale motors: biomedical opportunities and challenges," ACS Nano, vol. 6, no. 7, pp. 5745-5751, 2012.

[94] S. Hernot, S. Unnikrishnan, Z. Du et al., "Nanobodycoupled microbubbles as novel molecular tracer," Journal of Controlled Release, vol. 158, no. 2, pp. 346-353, 2012.

[95] R. Farra, N. F. Sheppard Jr., L. McCabe et al., "Firstin-human testing of a wirelessly controlled drug delivery microchip," Science Translational Medicine, vol. 4, no. 122, Article ID 122ra21, 2012.

[96] W. A. Qureshi, "Current and future applications of the capsule camera," Nature Reviews Drug Discovery, vol. 3, no. 5, pp. 447-450, 2004.

[97] P. C. Swain, "Wireless capsule endoscopy," Gut, vol. 52, supplement 4, pp. iv48-iv50, 2003. 
[98] D. G. Georganopoulou, L. Chang, J. M. Nam et al., "Nanoparticle-based detection in cerebral spinal fluid of a soluble pathogenic biomarker for Alzheimer's disease," Proceedings of the National Academy of Sciences of the United States of America, vol. 102, no. 7, pp. 2273-2276, 2005.

[99] J. M. Nam, K. J. Jang, and J. T. Groves, "Detection of proteins using a colorimetric bio-barcode assay," Nature Protocols, vol. 2, no. 6, pp. 1438-1444, 2007.

[100] J. B. M. Warntjes, O. Dahlqvist Leinhard, J. West, and P. Lundberg, "Rapid magnetic resonance quantification on the brain: optimization for clinical usage," Magnetic Resonance in Medicine, vol. 60, no. 2, pp. 320-329, 2008.

[101] A. J. Haes, L. Chang, W. L. Klein, and R. P. Van Duyne, "Detection of a biomarker for Alzheimer's disease from synthetic and clinical samples using a nanoscale optical biosensor," Journal of the American Chemical Society, vol. 127, no. 7, pp. 2264-2271, 2005.

[102] N. E. Kurland, Z. Drira, and V. K. Yadavalli, "Measurement of nanomechanical properties of biomolecules using atomic force microscopy," Micron, vol. 43, no. 2-3, pp. 116-128, 2012.

[103] V. Mani, B. V. Chikkaveeraiah, V. Patel, J. S. Gutkind, and J. F. Rusling, "Ultrasensitive immunosensor for cancer biomarker proteins using gold nanoparticle film electrodes and multienzyme-particle amplification," ACS Nano, vol. 3, no. 3, pp. 585-594, 2009.

[104] A. Neely, C. Perry, B. Varisli et al., "Ultrasensitive and highly selective detection of alzheimer's disease biomarker using two-photon rayleigh scattering properties of gold nanoparticle," ACS Nano, vol. 3, no. 9, pp. 2834-2840, 2009.

[105] Y. Heta, K. Kumaki, H. Hifumi, D. Citterio, A. Tanimoto, and K. Suzuki, "Gadolinium containing photochromic micelles as potential magnetic resonance imaging traceable drug carriers," Photochemistry and Photobiology, vol. 88, no. 4, pp. 876-883, 2012.

[106] J. M. J. Richards, C. A. Shaw, N. N. Lang et al., "In vivo mononuclear cell tracking using superparamagnetic particles of iron oxide feasibility and safety in humans," Circulation, vol. 5, no. 4, pp. 509-517, 2012.

[107] S. Metz, A. J. Beer, M. Settles et al., "Characterization of carotid artery plaques with USPIOenhanced MRI: assessment of inflammation and vascularity as in vivo imaging biomarkers for plaque vulnerability," International Journal of Cardiovascular Imaging, vol. 27, no. 6, pp. 901-912, 2011.

[108] D. Bataveljić, S. Stamenković, G. Bačić, and P. R. Andjus, "Imaging cellular markers of neuroinflammation in the brain of the rat model of amyotrophic lateral sclerosis," Acta Physiologica Hungarica, vol. 98, no. 1, pp. 27-31, 2011.

[109] L. MacHtoub, R. Pfeiffer, A. Backovic, S. Frischauf, and M. C. Wick, "Molecular imaging cellular SPIO uptake with nonlinear optical microscopy," Journal of Medical Imaging and Radiation Sciences, vol. 41, no. 3, pp. 159-164, 2010.

[110] S. Santra, S. D. Jativa, C. Kaittanis, G. Normand, J. Grimm, and J. M. Perez, "Gadolinium-encapsulating iron oxide nanoprobe as activatable NMR/MRI contrast agent," ACS Nano, vol. 6, no. 8, pp. 7281-7294, 2012.

[111] A. H. Jacobs and B. Tavitian, "Noninvasive molecular imaging of neuroinflammation," Journal of Cerebral Blood Flow and Metabolism, vol. 32, no. 7, pp. 1393-1415, 2012. 


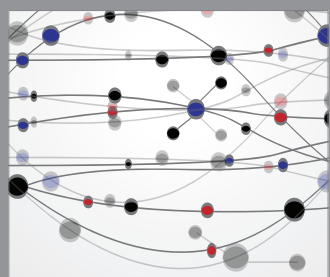

The Scientific World Journal
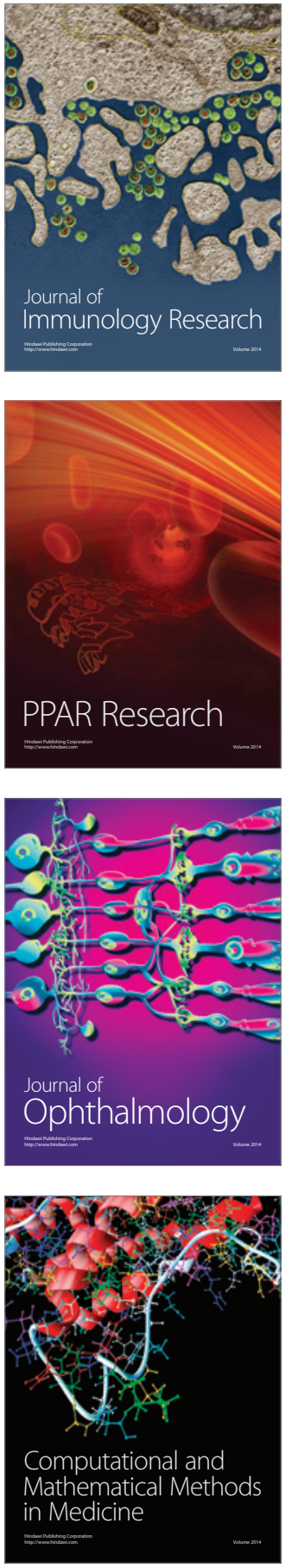

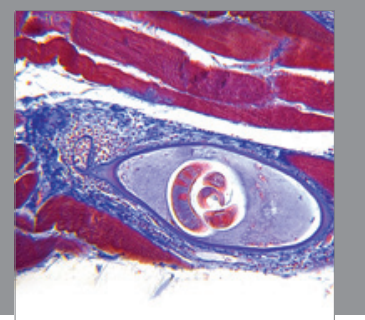

Gastroenterology

Research and Practice
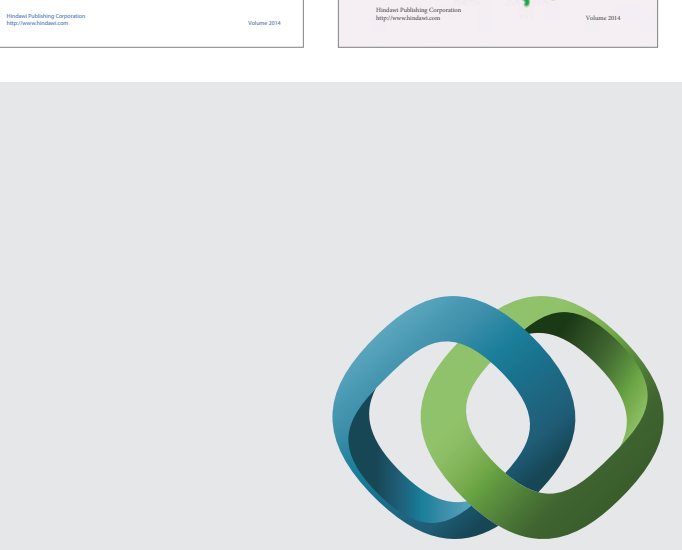

\section{Hindawi}

Submit your manuscripts at

http://www.hindawi.com
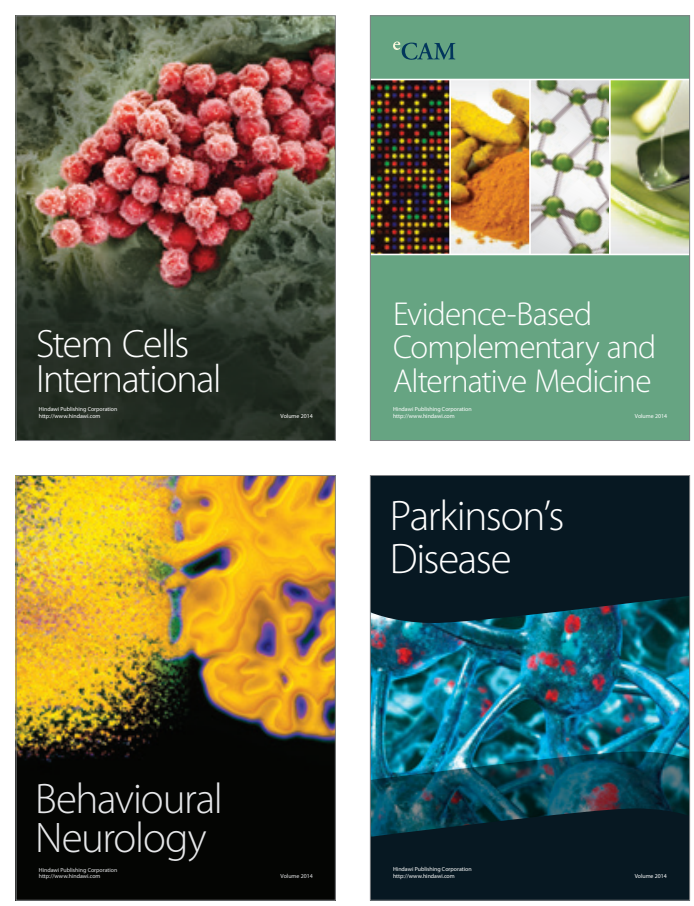

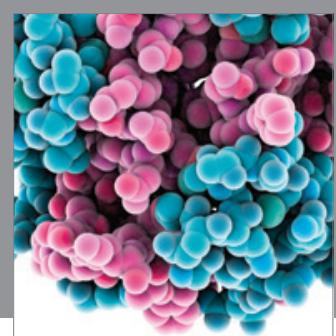

Journal of
Diabetes Research

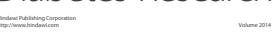

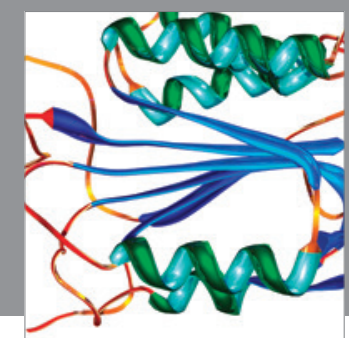

Disease Markers
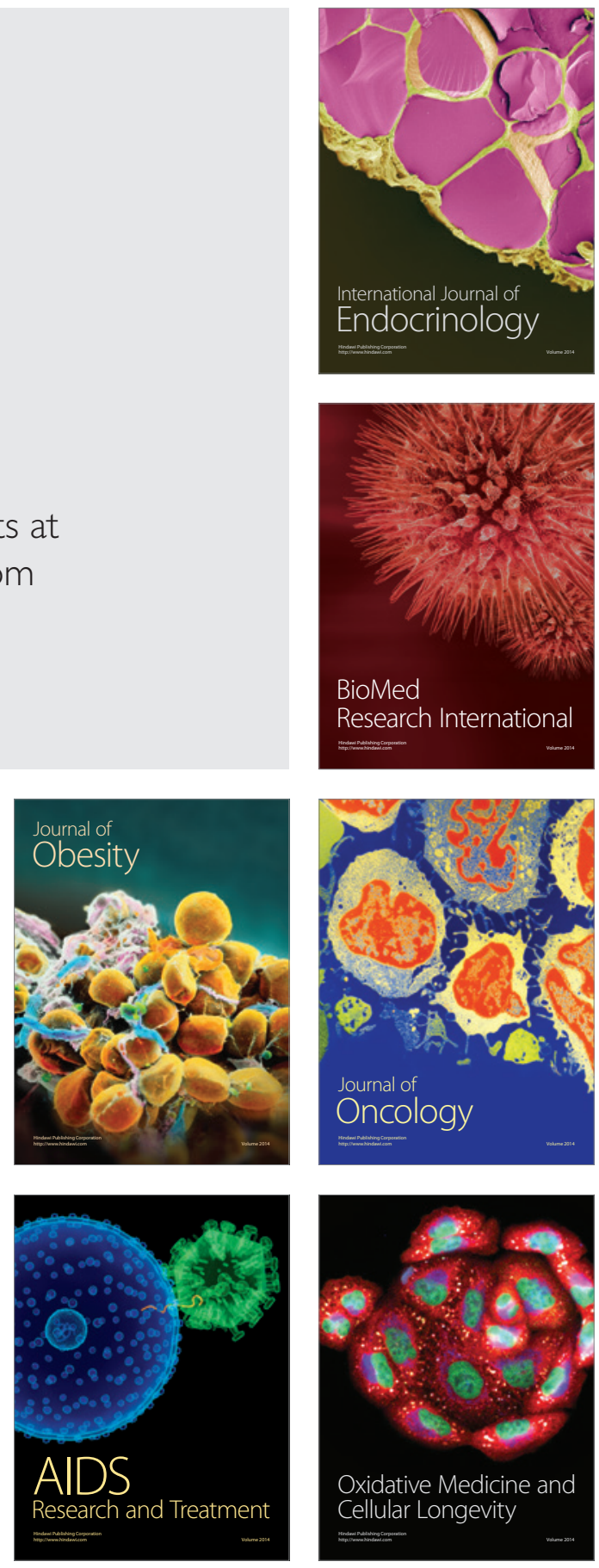